10.3

\title{
Применение корреляционного метода для определения количества вспыхивающих пузырьков и количества фотонов в вспышке при многопузырьковой сонолюминесценции
}

\author{
(C) М.В. Казачек, Т.В. Гордейчук \\ Тихоокеанский океанологический институт им. В.И. Ильичева ДВО РАН, Владивосток, Россия \\ ฯ E-mail: tanya@poi.dvo.ru
}

Поступило в Редакцию 25 июня 2019 г.

В окончательной редакции 13 декабря 2019 г.

Принято к публикации 13 декабря 2019 г.

\begin{abstract}
Методом счета корреляций между фотонами с использованием соотношений комбинаторики оценено количество вспыхивающих пузырьков за период ультразвука и количество фотонов, излучаемых пузырьком за вспышку при облучении водных растворов $\mathrm{NaCl}$ и додецилсульфата $\mathrm{Na}$ ультразвуком частоты $20 \mathrm{kHz}$. Количество вспыхивающих пузырьков $\sim 100$, что значительно меньше общего количества пузырьков в облаке и отражает значительную роль динамики пузырька для генерации вспышки. Количество фотонов от пузырька за вспышку 10000 , что на порядок меньше, чем при стабильной сонолюминесценции одиночного пузырька.
\end{abstract}

Ключевые слова: сонолюминесценция, число пузырьков, число фотонов, корреляционный метод.

DOI: 10.21883/PJTF.2020.06.49157.17946

Многопузырьковая сонолюминесценция (МПСЛ) сопровождает ультразвуковую кавитацию в жидкостях и происходит в виде коротких вспышек нелинейно пульсирующих пузырьков. В водных растворах, содержащих $\mathrm{Na}$, спектры сонолюминесценции включают широкий континуум и $D$-линию Na. Длительность вспышек этих спектральных компонент в ходе одного акта высвечивания оказывается различной. Полученные нами корреляционные спектры для МПСЛ позволили вычислить ширину и взаимный сдвиг вспышек континуума и натрия в пузырьковом облаке [1]. Помимо этого корреляционные спектры содержат информацию о количестве вспыхивающих пузырьков за один период ультразвука и среднем количестве фотонов, излучаемых пузырьком за вспышку. Задача интересна для исследования природы МПСЛ и динамики кавитационной области и решается разными методами [2-4]. Эти оценки являются целью настоящей работы. Сопоставление количества фотонов, излучаемых при стабильной сонолюминесценции одиночного пузырька (СЛОП), и количества фотонов, испускаемых пузырьком в облаке при МПСЛ, позволяет сравнить излучательную эффективность пузырьков в обоих случаях. Определение доли вспыхивающих пузырьков в облаке дает возможность оценить роль динамических процессов при генерации вспышек.

Ультразвуковая ячейка проточного типа [5] дополнена счетчиком корреляций [1]. Частота ультразвука $20 \mathrm{kHz}$, общая поглощаемая мощность $20 \mathrm{~W}$, температура растворов $10^{\circ} \mathrm{C}$. Мы исследовали МПСЛ от насыщенных $\mathrm{Ar}$ водных растворов $\mathrm{NaCl} 5 \mathrm{M}$ и $0.5 \mathrm{M}$, додецилсульфата $\mathrm{Na}$ (SDS) $5 \mathrm{mM}$. Принципиальная схема регистрации представлена на рис. 1. Поток фото- нов состоит из компактных пакетов, излученных при вспышках отдельных пузырьков. Для определения длительности вспышек был применен метод задержанных совпадений.

Нагрузкой ФЭУ служат сопротивления $\sim 50 \Omega$, подстраиваемые для согласования цепи. Регистрируемые однофотонные импульсы представляют отрицательные пики с крутым передним фронтом ( $1 \mathrm{~ns})$ и пологим задним ( 10 ns). Осциллограф RIGOL DS1104Z синхронно записывает отрезки сигналов $f_{1}(t)$ и $f_{2}(t)$ длительностью $M=60000$ точек и передает их в компьютер, время $t$ дискретно с шагом $2 \mathrm{~ns}$. Производятся многократное чтение и обработка отрезков сигналов с целью накопления корреляций между импульсами.

Назовем полными корреляционными функциями в дискретном случае

$$
k_{i j}(\tau)=\sum f_{i}(t) f_{j}(t+\tau),
$$

где $i, j=1,2-$ номера каналов, $\tau$ - время задержки, суммирование по $t$ проводится по всей длине отрезка сигнала $M$. Функций четыре (две авто- и две взаимно корреляционные), и они представляют распределения интервалов между импульсами в двух каналах по длительностям. Для измерения ширины вспышки $s_{h}$ и числа пузырьков в нашей схеме регистрации использовалась функция $k_{12}$. Синхронные фотоны образуют пик функции с шириной, пропорциональной ширине вспышки, который сдвинут от нуля линией задержки $\tau_{d}$ (DELAY на рис. 1). При $\tau_{d} \gg s_{h}$ весь пик функции укладывается в $k_{12}$. Для вычисления $k_{i j}(\tau)$ „в штуках“ произведение $f_{i} f_{j}$ полагается равным нулю, 


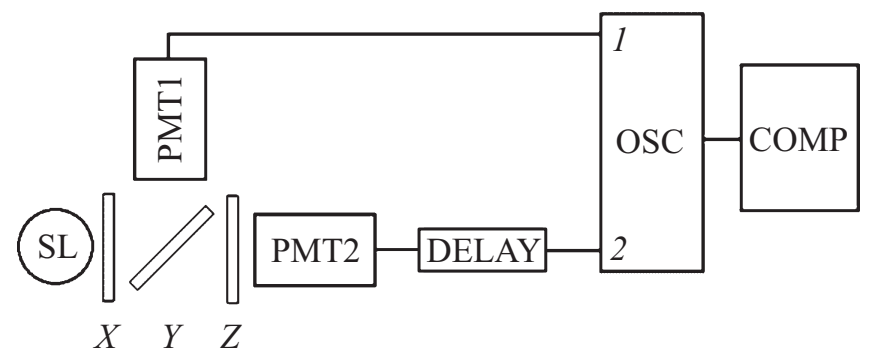

Рис. 1. Схема реализации метода задержанных совпадений (корреляционного метода) для МПСЛ (SL). PMT - фотоумножители, DELAY - линия задержки, OSC - цифровой осциллограф, СОМР - компьютер. $X, Y, Z-$ позиции для светофильтров.

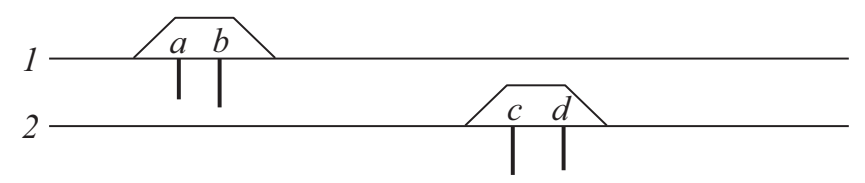

Рис. 2. Огибающие пакетов импульсов в двух каналах и фотонные импульсы $a, b, c, d$.

если импульсы не совпали, либо единице, если импульсы совпали. Момент прихода импульса регистрируется по прохождению передним фронтом сигнала заданного порога.

Назовем неполными корреляционными функциями

$$
k_{i j}(\tau)=\sum f_{i}(t) f_{j}(t+\tau),
$$

где суммирование проводится по значениям $t$, относящимся только к „соседним“ импульсам в каналах $i, j$. Мы считаем корреляции по алгоритму, основанному на (2), имеющему преимущество по скорости перед (1) за счет однократного прохождения массивов длины $M[1]$.

Различие между (1) и (2) покажем на примере, где в каждом пакете регистрируются два импульса. На рис. 2 показаны огибающие пакетов импульсов в двух каналах (в реальном эксперименте они ненаблюдаемы) и сами фотонные импульсы $a, b, c, d$. В полной функции $k_{12}(1)$ будут учтены четыре интервала между импульсами: $a c, a d, b c, b d$, в неполной функции $k_{12}(2)$ - только один интервал между соседними импульсами $b c$. Как показал численный эксперимент, это приводит к деформации корреляционной функции (2) относительно (1), если среднее число импульсов на пакет больше единицы. В реальном эксперименте частота счета импульсов менее $100 \mathrm{kHz}$, и мы регистрируем в среднем менее пяти импульсов за период ультразвука. Эти импульсы излучены облаком из сотен пузырьков. Это означает, что от пузырька за период в среднем регистрируется много меньше одного фотона, и мы вправе применить способ (2).
На рис. 3 приведены ненормированные корреляционные функции $k_{12}$ и $k_{21}$, полученные от МПСЛ водного $\mathrm{NaCl} 5 \mathrm{M}$. Здесь в позицию $X$ устанавливался оранжевый фильтр для выделения излучения $\mathrm{Na}$ или фиолетовый фильтр для выделения излучения континуума (cont), в позицию $Y$ - нейтральный (рис. 1). В каналы 1 и 2 поступали импульсы одного типа, их корреляции мы обозначим как $\mathrm{Na}-\mathrm{Na}$ и cont-cont. Корреляционный пик $k_{12}$ для $\mathrm{Na}$ показан на рис. $3, a$, для континуума на рис. $3, c$. На рис. $3, b, d$ приведены те же функции в большем масштабе времени. Данные для МПСЛ $\mathrm{NaCl} 0.5 \mathrm{M}$ и SDS $5 \mathrm{mM}$ выглядят подобно приведенным на рис. 3.

Пусть в облаке за период ультразвука $T$ однократно вспыхивают $m$ пузырьков, каждый излучает $n$ фотонов, всего $m n$ фотонов. Корреляция - набор из двух фотонов. Вероятность того, что два фотона придут от одного и того же пузырька, можно определить из числа сочетаний. Общее число событий „взяты два фотона“ равно числу сочетаний по 2 из $m n$

$$
C_{m n}^{2}=m n ! / 2 ! /(m n-2) !=m n(m n-1) / 2 .
$$

Число событий „два фотона из одного пузырька“ равно $C_{n}^{2}=n ! / 2 ! /(n-2) !=n(n-1) / 2$, число событий ,два фотона из одного любого пузырька“ в $m$ раз больше:

$$
m C_{n}^{2}=m n(n-1) / 2
$$

Вероятность того, что два фотона окажутся из одного и того же пузырька, равна отношению числа событий (4) и (3): $P_{1}=m C_{n}^{2} / C_{m n}^{2}=(n-1) /(m n-1)$. Принимая, что $m \gg 1$ и $n \gg 1$, получаем $P_{1} \approx n /(m n)=1 / m$, т.е. она не зависит от числа фотонов от пузырька. Вероятность противоположного события (того, что два фотона пришли от разных пузырьков) $P_{2}=1-1 / m$. С учетом того, что $m \gg 1$, $P_{2} \approx 1$.

Рассмотрим, как распределены эти события по временно́й шкале задержек (интервалов). Корреляции между фотонами, приходящими от одного пузырька, $K_{1}$ сосредоточены в интервале времени вспышки. Назовем их „короткие“ корреляции. Корреляции между фотонами, приходящими от разных пузырьков, $K_{2}$ рассредоточены по периоду ультразвука $T$. Назовем их ,длинные“ корреляции. Будем считать, что вспышки случайны и заполняют половину периода ультразвука в соответствии c [6].

По числовым данным, использованным для построения рис. 3, $a$ (для эмиссии $\mathrm{Na}$ при МПСЛ $\mathrm{NaCl} 5 \mathrm{M}$ ), просуммируем число коротких корреляций, образующих пик, по $n_{0}=12$ точкам, $K_{1}=698$. Это число завышено на фон длинных корреляций, что будет учтено далее. Оценим число длинных корреляций $K_{2}$. Как видно из рис. $3, b, d$ в большем масштабе времен задержек, $k_{12}$ и $k_{21}$ спадают в пределе до нуля. Это 

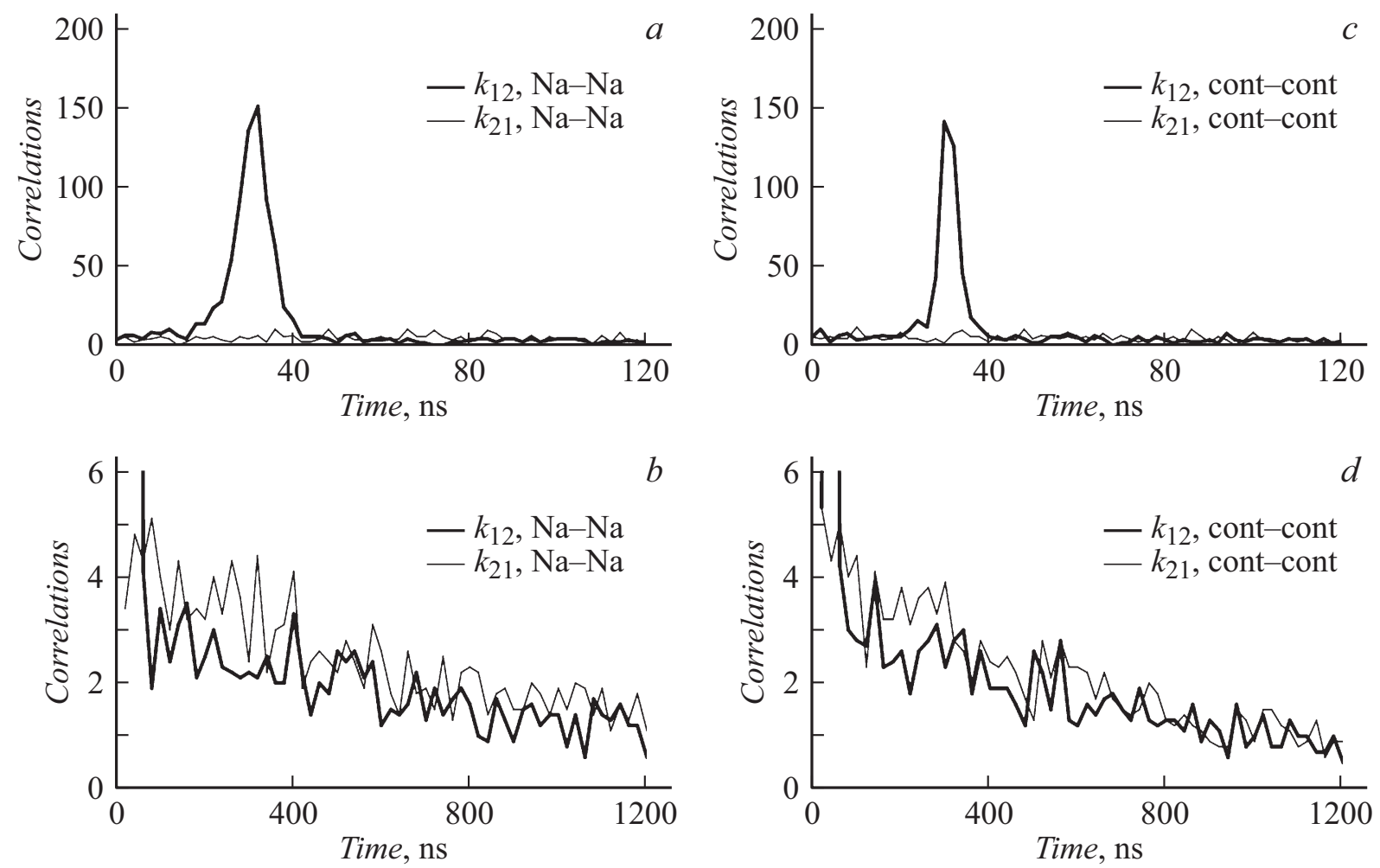

Рис. 3. Корреляционные функции $k_{12}$ и $k_{21}$ от фотонных импульсов МПСЛ $\mathrm{NaCl} 5 \mathrm{M}$ в двух масштабах времени. $a, b-$ корреляции $\mathrm{Na}-\mathrm{Na} ; c, d-$ корреляции cont-cont.

следствие эффекта „экранировки“ в алгоритме (2), который учитывает только соседние интервалы. Математически распределение соседних интервалов по длинам должно спадать экспоненциально. Правильным будет взять начальный уровень корреляционной функции $k_{12}$ в области, например, 0-100 ns (50 точек), исключая область пика, и распространить его на половину периода $T / 2=25000 \mathrm{~ns}$. Сумма корреляций $k_{12}$ вне пика по $n_{1}=38$ точкам $\left(n_{0}+n_{1}=50\right) k_{1}=144$. Для повышения точности используем дополнительно функцию $k_{21}$, которая имеет такой же вид, за исключением пика. Сумма корреляций по $n_{2}=50$ точкам $k_{2}=216$. Среднее число таких корреляций на точку будет равно $p=\left(k_{1}+k_{2}\right) /\left(n_{1}+n_{2}\right)=4.09$. Таким образом, мы получим $K_{2}=p / 2 \cdot T / 2 \approx 51000$. Отсюда же поправка для $K_{1}=698-n_{0} p \approx 649$.

Итак, мы получили число коротких корреляций, относящихся к одному и тому же пузырьку, и число длинных корреляций, относящихся к разным пузырькам. Их отношение должно равняться отношению вероятностей $P_{1}$ и $P_{2}$, найденных выше:

$$
K_{2} / K_{1}=P_{2} / P_{1}=m
$$

Для нашего случая $m(\mathrm{Na}) \approx 79$ пузырьков, дающих вспышки $\mathrm{Na}$ при МПСЛ $\mathrm{NaCl} 5 \mathrm{M}$, что получено в рамках принятых допущений без учета статистики пузырьков. Замечательно, что для оценки $m$ не потребо- валось учитывать долю светового потока, достигающего счетчика корреляций.

Аналогично (5) находим число пузырьков, излучающих континуум при МПСЛ $\mathrm{NaCl} 5 \mathrm{M}$, используя числовые данные для рис. $3, c, d, m($ cont $) \approx 142$. Для $\mathrm{NaCl} 0.5 \mathrm{M}$ получаем $m(\mathrm{Na}) \approx 23, m($ cont $) \approx 35$. Для SDS $5 \mathrm{mM} m(\mathrm{Na}) \approx 73, m($ cont $) \approx 60$.

Полное число фотонов можно оценить по количеству фотонных импульсов, приходящих на счетчик. Пусть за $L$ отрезков сигнала длиной $M=60000$ точек $(1$ точка $=2 \mathrm{~ns})$ в канале накоплено $P$ импульсов. Эффективное время накопления $2 L M[\mathrm{~ns}]$. Число принятых импульсов $N$ за период ультразвука $T=50000 \mathrm{~ns}$ будет равно

$$
N=P T /(2 L M) \text {. }
$$

Чтобы узнать число излученных за период фотонов $m n$, нужно учесть апертуру наблюдения $A$, квантовую эффективность фотокатода $Q$, оптические потери $S$ :

$$
N=m n A Q S
$$

Апертуру найдем из геометрических размеров нашей экспериментальной установки. Облако пузырьков находится на глубине $10 \mathrm{~cm}$ от выходного окна, еще $10 \mathrm{~cm}$ отделяют его от фотокатода. Диаметр фотокатода $2 \mathrm{~cm}$, телесный угол, вырезаемый им, равен $\Omega=\pi r^{2} / R^{2}, r=1 \mathrm{~cm}, R=20 \mathrm{~cm}$. Полный телесный 
Число фотонов от пузырька за вспышку согласно (8)

\begin{tabular}{c|c|c|r|c|c|c}
\hline \multirow{2}{*}{ Канал } & \multicolumn{2}{|c|}{$\mathrm{NaCl} 5 \mathrm{M}$} & \multicolumn{2}{c|}{$\mathrm{NaCl} 0.5 \mathrm{M}$} & \multicolumn{2}{c}{$\mathrm{SDS} 5 \mathrm{mM}$} \\
\cline { 2 - 7 } & $\mathrm{Na}$ & $\mathrm{cont}$ & $\mathrm{Na}$ & $\mathrm{cont}$ & $\mathrm{Na}$ & $\mathrm{cont}$ \\
\hline 1 & 4938 & 57452 & 10053 & 19928 & 10058 & 14184 \\
2 & 2061 & 69941 & 4559 & 14981 & 4192 & 13168 \\
Среднее & 3500 & 63700 & 7300 & 17500 & 7100 & 13700
\end{tabular}

угол равен $\Omega_{0}=4 \pi$, отсюда $A=\Omega / \Omega_{0}=1 / 1600$. Более точный учет геометрии дает для двух каналов $A(1)=0.0005, A(2)=0.001$. Квантовая эффективность фотокатода (из справочника) $Q \approx 0.15$ в максимуме спектральной чувствительности ФЭУ (400-500 nm). Эта область соответствует максимуму спектра континуума. В области линии $\mathrm{Na}(590 \mathrm{~nm})$ относительная спектральная чувствительность ФЭУ падает с 1 до 0.4 , поэтому примем $Q$ (cont) $\approx 0.15, Q(\mathrm{Na}) \approx 0.06$. Оптические потери различны в каналах и зависят от условий эксперимента (рис. 1). При измерении корреляций $\mathrm{Na}-\mathrm{Na}$ в позиции $X$ установлен оранжевый фильтр, при измерении корреляций cont-cont в позиции $X$ установлен фиолетовый фильтр. Для $\mathrm{NaCl} 5 \mathrm{M}$ при измерении корреляций cont-cont рядом с ним добавлен фильтр-сетка, пропускающий 0.12 часть падающего светового потока. Исходя из характеристик фильтров и спектров МПСЛ мы рассчитали, что оранжевый фильтр пропускает 0.85 излучения $\mathrm{Na}$, а фиолетовый - 0.1 излучения континуума в области 200-600 nm. В позиции $Y$ установлен нейтральный фильтр-делитель, коэффициент отражения (на канал 1) 0.15, коэффициент пропускания (на канал 2) 0.85. В позиции $Z$ при измерении континуума установлен фильтр-сетка, пропускающий 0.45. Кроме этого в канале 1 есть еще четыpe оптические границы раздела, пропускающие 0.72 , в канале 2 - две границы раздела, пропускающие 0.85 . Итоговая формула для нахождения $n$ из (6) и (7) имеет вид

$$
n=P T /(2 L M m A Q S)=5 P /(12 L m A Q S),
$$

где все множители зависят от конкретного эксперимента. Результаты представлены в таблице.

По нашим оценкам число вспыхивающих пузырьков, излучающих $\mathrm{Na}$ и/или континуум, за период ультразвука менее или около 100. Это неожиданно мало, так как МПСЛ можно видеть как густое облако вспышек, сияние. Но глаз воспринимает много периодов. Плотность пузырьков в облаке МПСЛ в воде $\sim 10^{4} \mathrm{~cm}^{-3}[7,8]$. В объеме рассматриваемой ячейки следует ожидать $\sim 10^{5}$ пузырьков. Это означает, что только малая (1/1000) часть пузырьков в облаке участвует в МПСЛ и что пузырек может вспыхивать далеко не каждый период, а только когда его динамические характеристики позволяют достигнуть условий для вспышки.
Количество пузырьков, излучающих $\mathrm{Na}$ и континуум, оказывается различным, возможно вследствие разного распределения вспышек $\mathrm{Na}$ и континуума по периоду. Кроме того, это может отражать деление пузырьков на сонохимические и сонолюминесцентные [9]. Количество вспыхивающих пузырьков различается по растворам: в $\mathrm{NaCl} 0.5 \mathrm{M}$ примерно вдвое меньше, чем в $\mathrm{NaCl} 5 \mathrm{M}$ и SDS $5 \mathrm{mM}$. Это различие коррелирует с общей интенсивностью МПСЛ и не коррелирует, например, с шириной спектральной линии $\mathrm{Na}[10]$.

Число фотонов за период ультразвука при СЛОП $\sim 10^{5}[11,12]$. Для МПСЛ горячих паровых пузырьков число излучаемых пузырьком фотонов $\sim 10^{3}$ [13]. В нашем случае число фотонов от пузырька за вспышку оценено $\sim 10^{4}$ (см. таблицу). Число фотонов $\mathrm{Na}$ в $\sim 2-20$ раз меньше числа фотонов континуума. Эффективность генерации фотонов при МПСЛ таким образом в 10 раз ниже, чем при СЛОП.

В работе предложен эффективный способ оценки числа вспыхивающих пузырьков за период ультразвука при МПСЛ методом счета корреляций. Определено среднее число фотонов, излучаемых пузырьком за вспышку в пузырьковом облаке.

Дополнительные материалы к этой статье доступны в электронном виде (см. переводную версию).

\section{Финансирование работы}

Работа выполнена в рамках госбуджетной темы (регистрационный номер АААА-А17-117030110031-6).

\section{Конфликт интересов}

Авторы заявляют, что у них нет конфликта интересов.

\section{Список литературы}

[1] Казачек М.В., Гордейчук Т.В. // Приборы и техника эксперимента. 2019. № 1. С. 28-29. DOI: $10.1134 / \mathrm{S} 0032816219010117$

[2] Cairós C., Mettin R. // Phys. Rev. Lett. 2017. V. 118. P. 064301 (1-5). DOI: 10.1103/PhysRevLett.118.064301

[3] Pflieger R., Nikitenko S.I., Ashokkumar M. // Ultrason. Sonochem. 2019. V. 59. P. 104753 (1-8). https://doi.org/10.1016/j.ultsonch.2019.104753

[4] Merouani S., Ferkous H., Hamdaoui O., Rezguic Y., Gueminic M. // Ultrason. Sonochem. 2015. V. 23. P. 37-45. http://dx.doi.org/10.1016/j.ultsonch.2014.09.009

[5] Гордейчук Т.В., Казачек М.В. // ЖФХ. 2019. Т. 93. № 5. C. 793-796. DOI: 10.1134/S004445371905011X

[6] Abe S., Choi P.K. // Jpn. J. Appl. Phys. 2009. V. 48. P. 07 GH02 (1-3). DOI: 10.1143/JJAP.48.07GH02

[7] Акуличев B.A. // Мощные ультразвуковые поля / Под ред. Л.Д. Розенберга М: Наука, 1968. С. 129-166.

[8] Didenko Y.T., Pugach S.P. // J. Phys. Chem. 1994. V. 98. P. 9742-9749. DOI: 10.1021/j100090a006

[9] Brotchie A., Grieser F., Ashokkumar M. // Phys. Rev. Lett. 2009. V. 102. P. $084302(1-4)$. DOI: 10.1103/PhysRevLett.102.084302 
[10] Казачек М.В., Гордейчук Т.В. // Письма в ЖТФ. 2011. T. 37. B. 6. C. 39-48. DOI: $10.1134 / \mathrm{S} 1063785011030242$

[11] Gompf B., Gunther R., Nick G., Pecha R., Eisenmenger W. // Phys. Rev. Lett. 1997. V. 79. P. 1405-1408. DOI: 10.1103/PhysRevLett.79.1405

[12] Didenko Y.T., Suslick K.S. // Nature. 2002. V. 418. P. 394-397. DOI: $10.1038 /$ nature00895

[13] Chakravarty A., Walton A.J. // J. Lumin. 2001. V. 92. P. 2733. 